\title{
Visual Evoked Potential as a Clinical Tool to Evaluate Changes in Brain Function Associated with Concussion
}

ISSN: 2576-8875

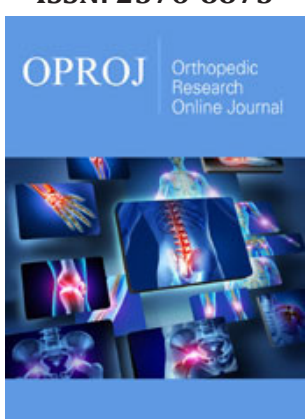

${ }^{* 1}$ Corresponding author: Robert A Orsillo, Orsillo Vision Care and Optical, Tallahassee, FL, USA

Submission: 留June 12, 2019

Published: 沮June 21, 2019

Volume 5 - Issue 5

How to cite this article: Robert $A$ Orsillo, Prashant S, Angela S, Peter D. Visual Evoked Potential as a Clinical Tool to Evaluate Changes in Brain Function Associated with Concussion. Ortho Res Online J. 5(5). OPROJ.000621.2019. DOI: 10.31031/OPROJ.2019.05.000621

Copyright@: Robert A Orsillo, This article is distributed under the terms of the Creative Commons Attribution 4.0 International License, which permits unrestricted use and redistribution provided that the original author and source are credited.

\author{
Robert A Orsillo ${ }^{1 *}$, Prashant Singh ${ }^{2}$, Angela Sehgal ${ }^{2}$ and Peter Derr ${ }^{3}$ \\ ${ }^{1}$ Orsillo Vision Care and Optical, Tallahassee, FL, USA \\ ${ }^{2}$ Nutrition, Food \& Exercise Sciences, Florida State University, Tallahassee, FL, USA \\ ${ }^{3}$ Diopsys, Vision Systems Development, Electrophysiology, Pine Brook, NJ, USA
}

\begin{abstract}
Significance: Concussions or mild traumatic brain injuries (mTBIs) are the most common form of traumatic brain injury. There is lack of reliable, diagnostic tools in healthcare for the treatment of mTBI. A Visual Evoked Potential (VEP) approach will enhance practitioners in diagnosing and treating mTBI.

Purpose: The aim of this study was to evaluate changes in brain function associated mTBI/post-concussion syndrome (PCS) using Visual Evoked Potential (VEP). We examined the effects of mTBI on early visual processing to determine if the signs and symptoms of mTBI reflected the failure of retinal signal latency based on the paradigm that low spatial frequency (LSF) are processed faster than high spatial frequency (HSF) when measured at the primary visual cortex.
\end{abstract}

Methods: The VEP of participants were measured by biasing the magnocellular pathways using a low Michelson contrast, temporally modulated phase reversing checkerboard stimulus pattern, and successive spatial frequency (SF) from LSF to increasing HSF. The VEP values of participants from the control group where compared with VEP values obtained from the mTBI/ PCS group. Participants from the mTBI/PCS group underwent a 6 week-7 month treatment

Results: The mTBI/PCS group failed to process the LSF faster than the increasing HSF, which resulted in loss of top-down information that leads to meaningful perception.

Conclusions: The mTBI/PCS subjects resolved their VEP deficits and returned to the proper temporal organization of latency, compared with the results from the control group.

Keywords: Concussion; mTBI; Post-concussion syndrome; Spatial frequency; Thalamus; Vision therapy

Introduction

Concussions remain the most common form of Traumatic Brain Injury (TBI) in the world today. This traumatic event occurs when two shock waves pass through the brain resulting in bruising of the underlying cortex. Subsequently, additional acceleration/deceleration forces result in stretching or twisting, and rotational acceleration forces lead to the stretching and shearing of neural and glia components resulting in a diffuse axonal injury (DAI) [1]. Synaptic disruption, resulting from the onset of a neuro-chemical cascade, then initiates metabolic and pathophysiological disruptions of brain function $[2,3]$.

The American Congress of Rehabilitation Medicine has stated that concussions occur when any one of the following the functional manifestations occur: "any period of loss of consciousness, loss of memory for events immediately before or after an accident, any alteration of mental state (feeling dazed, disoriented, or confused), and focal neurological deficits that may or may not be transient". Other indications of a concussion include "loss of consciousness for 30 minutes or less, an initial Glascow Coma Scale (GCS) of 13-15, and/ or posttraumatic amnesia not greater than 24 hours" (American Congress of Rehabilitation Medicine, 1993). If one of these manifestations occurred, then the condition is classified as 
mild Traumatic Brain Injuries (mTBI) [4]. The widely accepted understanding of mTBIs is the absence of any structural damage when observed by traditional neuro-imaging techniques e.g. CT scan, X-ray, MRI. Among healthcare providers it is generally understood that a mTBI is not a very serious injury, with only transient disturbances, which typically resolve in several days to weeks and requires only observation [5].

Concussion complications are manifested by a combination of specific and non-specific symptoms that may include, headache, dizziness, light sensitivity, fatigue, mental fog, orientation problems, sleep disturbance, dizziness, and loss of balance [5,6]. The symptoms of a mTBI have been associated with the Magnocellular (M), and dorsal stream pathway processing. In addition to the symptoms, other signs include oculomotor dysfunction, such as convergence insufficiency, poor fusional reserves, accommodative disturbances, saccadic and pursuit movement disorders [7]. If these signs and symptoms continue long after the expected recovery period (estimated as anywhere from 10 days to 3 months) this is classified as Post-Concussion Syndrome (PCS). Unlike mTBIs, there seems to be a lack of consensus regarding the definition of PCS $[5,8]$.

Increasing evidence supports a thalamic hypothesis as the central mechanism for global cognitive impairment from mTBI/ PCS [9]. King et al. [10] examined brain displacement and deformation using various concussive forces on cadavers. Their findings indicated a primary injury to the thalamus. In addition, Little et al. [11] discovered damages to cortical-subcortical fibers projecting to and from the thalamus, which contributed to chronic impairment in cognition and behavior, otherwise known as a secondary injury.

Except for the most severe brain injury cases, function resumed because neural connections create new networks, which can by-pass the damaged connections. Utilizing a top - down visual therapy program with recruitment from cortical areas of the brain will assist in the recovery of function and elimination of mTBI/PCS symptoms among subjects. Chang et al. described that for vision therapy to be effective, motivation, feedback, repetition, sensorymotor mismatch and multi-sensory integration are necessary components to enhance neuroplasticity changes [6].

Neuropsychological testing is the commonly used approach for evaluating the signs and symptoms of mTBI/PCS. A very widely used neuropsychological test tool is the ImPact test. Resch et al. [12] reported variation in test-retest reliability for ImPact metrics. Their data suggested that a multi-faceted approach is better for concussion assessment. Other evaluation tools for mTBI/PCS include neurological testing, structural imaging, and blood tests looking for cellular factors, with diminutive emphasis on functional electrodiagnostic testing.

Numerous investigations have recognized specific cellular factors in the blood as potential biomarkers for the detection of mTBI [13]. In February 2018 The US Food and Drug Administration (FDA) approved a blood test to aid with concussion evaluation in adults [14]. Known as Banyan BTI (Brain Trauma Indicator), the test measures levels of two protein biomarkers -- ubiquitin carboxy-terminal hydrolase-L1 and glial fibrillary acidic protein. This testing aims to reduce unnecessary radiation exposure from imaging to 'ensure that each patient is receiving the right imaging exam, at the right time, with the right radiation dose [15]. Diffusion Tensor Imaging (DTI), the preferred imaging technique, is used to evaluate the microscopic changes associated with DAI and glia disruption [16-18]. However, neuro-imaging does not show the dysfunctional effects or after effects of the neurochemical cascade; this test also does not reveal the extent of mitochondrial damage because of the injury [3].

The Visual Evoked Potential (VEP) test represents the response of the visual cortex to stimuli presented in the visual field. It is a very commonly used clinical test for ruling out disorders associated with the visual pathways. The VEP uses spatial frequency analysis to determine the stability of the afferent visual pathway. The aim of this study was to present VEP testing as an added clinical tool aimed to help fill the gaps in mTBI/PCS testing.

\section{Material and Methods}

The subject's M pathways were biased with temporally modulated phase reversing checkerboard stimulus pattern using successive spatial frequencies (SF) while simultaneously measuring the VEPs waveform parameters as an indicator of the bioelectric signal temporal order. Using the VEP to evaluate the retinal signal of the M pathway through the thalamus lateral geniculate nucleus (LGN) to the visual cortex, the researchers used a low contrast checkerboard pattern reversal stimuli, which created the subjective impression that the squares of the stimulus 'stream' across the screen as though they were moving [19].

\section{Participants}

The control group consisted of fifty-four participants ranging in age from 13-66 years old (Figure 1A: 33 males and 21 females). The control group participants were required to have no history of brain injury, neurological disease or any history of medications or substances that can affect the VEP. Each underwent a comprehensive visual examination including oculomotor binocular vision assessment and retinal evaluation with visual field. The participants were also tested with the Diopsys NOVA Vision Testing Systems VEP ad hoc module to measure their electrophysiological visual function response to successively presented SF of $16 \times 16$, $32 \times 32,64 \times 64$ checkerboard stimuli at a 15\% Michelson contrast level, with pattern reversals. Fifty-two mTBI/PCS patients ranging in age from 13-74 years old (Figure 1B: 32 males and 20 females) comprised the experimental group. All subjects underwent comprehensive visual examination identical to the control group and were also tested with Diopsys NOVA Vision Testing Systems VEP ad hoc module to measure their electrophysiological visual function response identical to the normal group. The treatment group consisted of 27 patients (Figure 1C:13 males and 14 females). All subjects in the treatment group received vision therapy, as described below in section. 

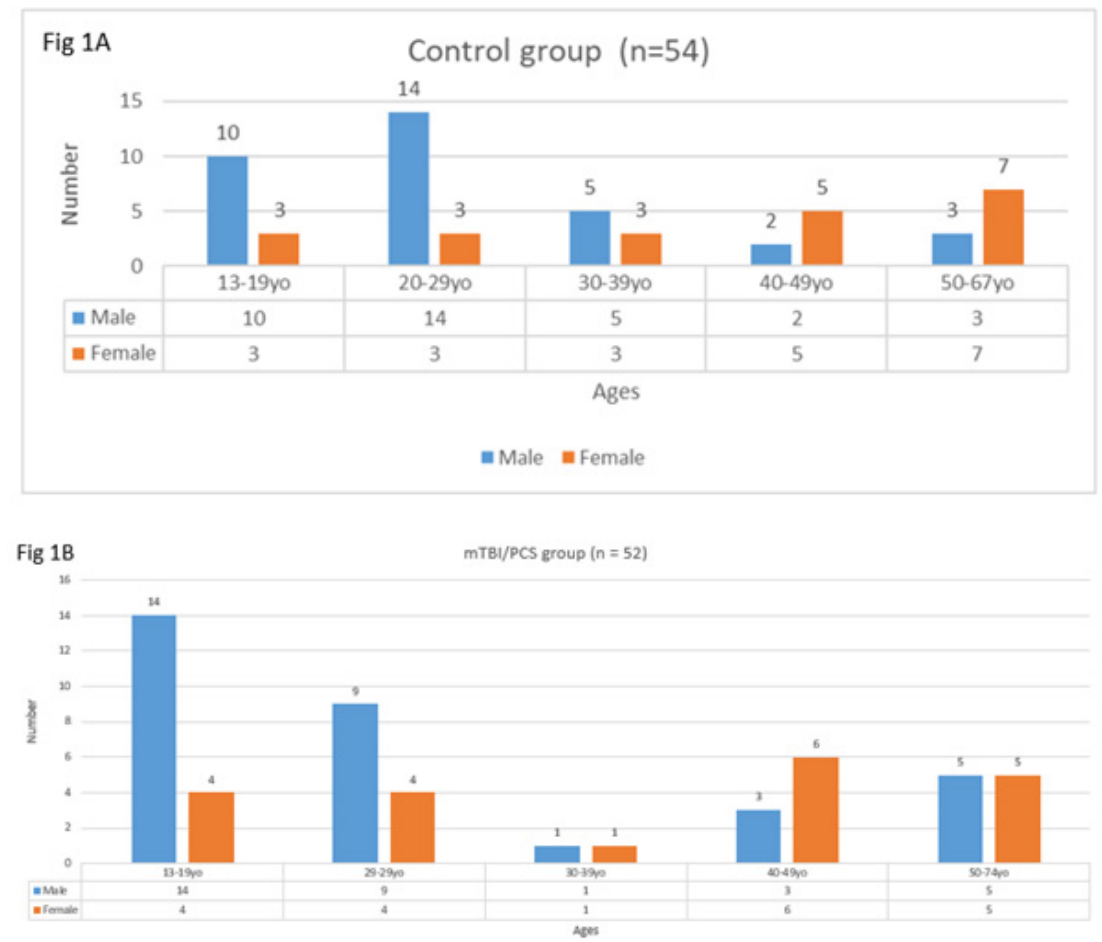

aner atement

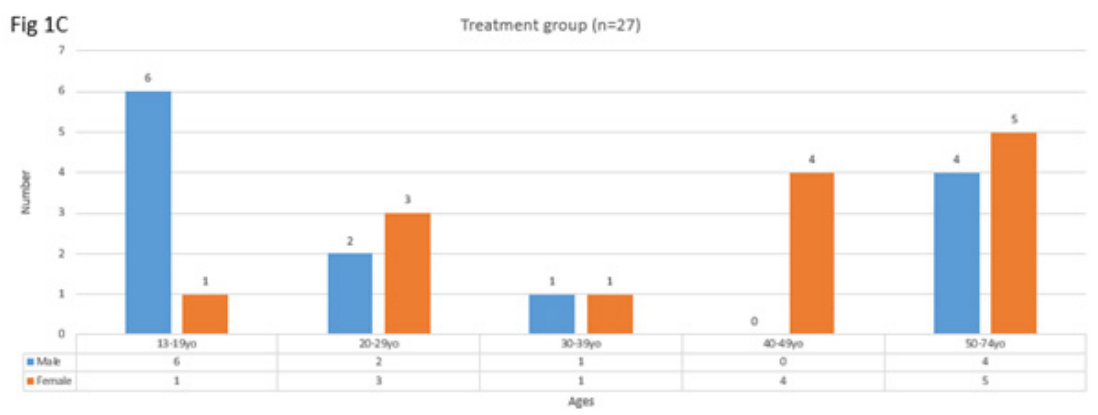

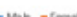

Figure 1: (1A) Distribution of control group by age and gender. (1B) Distribution of mTBI/PCS group by age and gender. (1C) Distribution of treatment group by age and gender that received vision therapy.

\section{Vision therapy}

The vision therapy treatment protocol administered to the experimental group included multi-sensory training components. The vision therapy program lasted from 6 weeks to 7 months depending on the onset and severity of signs and symptoms among the experimental participants. The components of the program exercises included "Top Down Processing" programming as described by Cohen and Chang [6], random stimulus movement from saccadic to smooth ocular motion, ocular-vestibular integration with a head sensor feedback, proprioceptive balance board movements with vibration cues, eye fixation biofeedback monitoring, and vestibular sway detection during all activity.

\section{Statistical analysis}

Microsoft Excel ${ }^{\circledR}$ spreadsheet software was used to plot participant distribution and to compute confidence intervals of mean latencies.

\section{Results}

The control group VEP values exhibited a temporal order of organization of subsequent neural impulses to visual cortex indicated by an upward slope (Figure 2A, 2B). The VEP results for the $\mathrm{mTBI} / \mathrm{PCS}$ group showed a disorganization of subsequent neural impulses resulting in a skewed, non-upward slope. This skewed, non-upward slope observed in the mTBI/PCS can be interpreted as temporal disorder of the subsequent retinal impulse to the visual cortex (Figure 3A, 3B). Upon completion of their vision therapy, the treatment group was re-tested, the data was compared to their original baseline. The VEP results after vision therapy showed a return to normal temporal order of the subsequent increasing SF (Figure 4) among mTBI/PCS subjects.

Results obtained in this study showed reproducible VEP indications of dysfunction associated with SF processing and its correlation with the signs and symptoms associated with mTBI. 


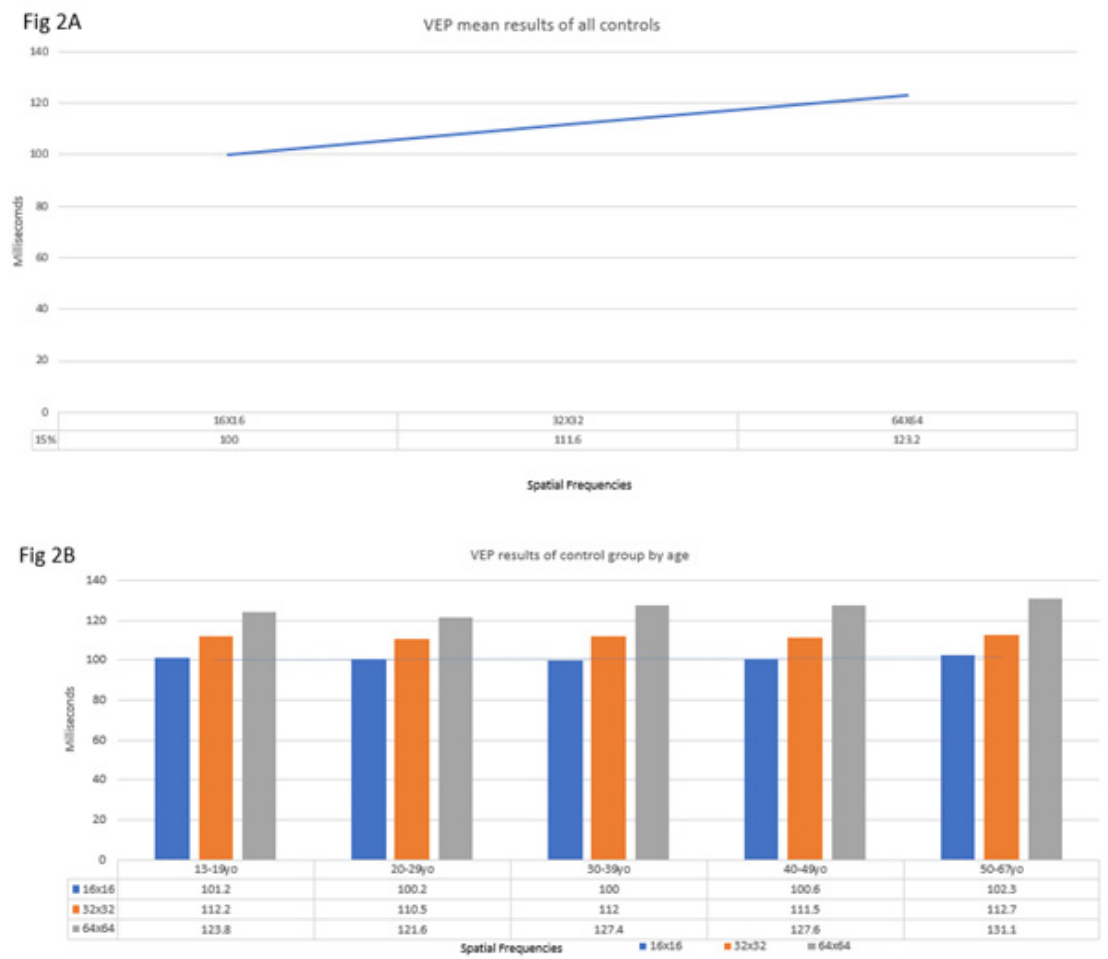

Figure 2: (2A) Upward slope interpreted as synchronization of the retinas neural network. The normal controls mean P100 latencies was $100 \mathrm{~ms}(95 \% \mathrm{CI}$ : $83 \mathrm{~ms}$ to $118 \mathrm{~ms}), 111.6 \mathrm{~ms}(95 \% \mathrm{CI}: 91.8 \mathrm{~ms}$ to $122 \mathrm{~ms}) 123.2 \mathrm{~ms}(95 \% \mathrm{CI}$ : $114 \mathrm{~ms}$ to $135 \mathrm{~ms}$ ) for the successive spatial frequencies. This upward slope is interpreted as synchronization of the retinas neural network. (2B) Mean results of each age group indicated an upward slope for the successive spatial frequencies designated synchronization for the various age group.
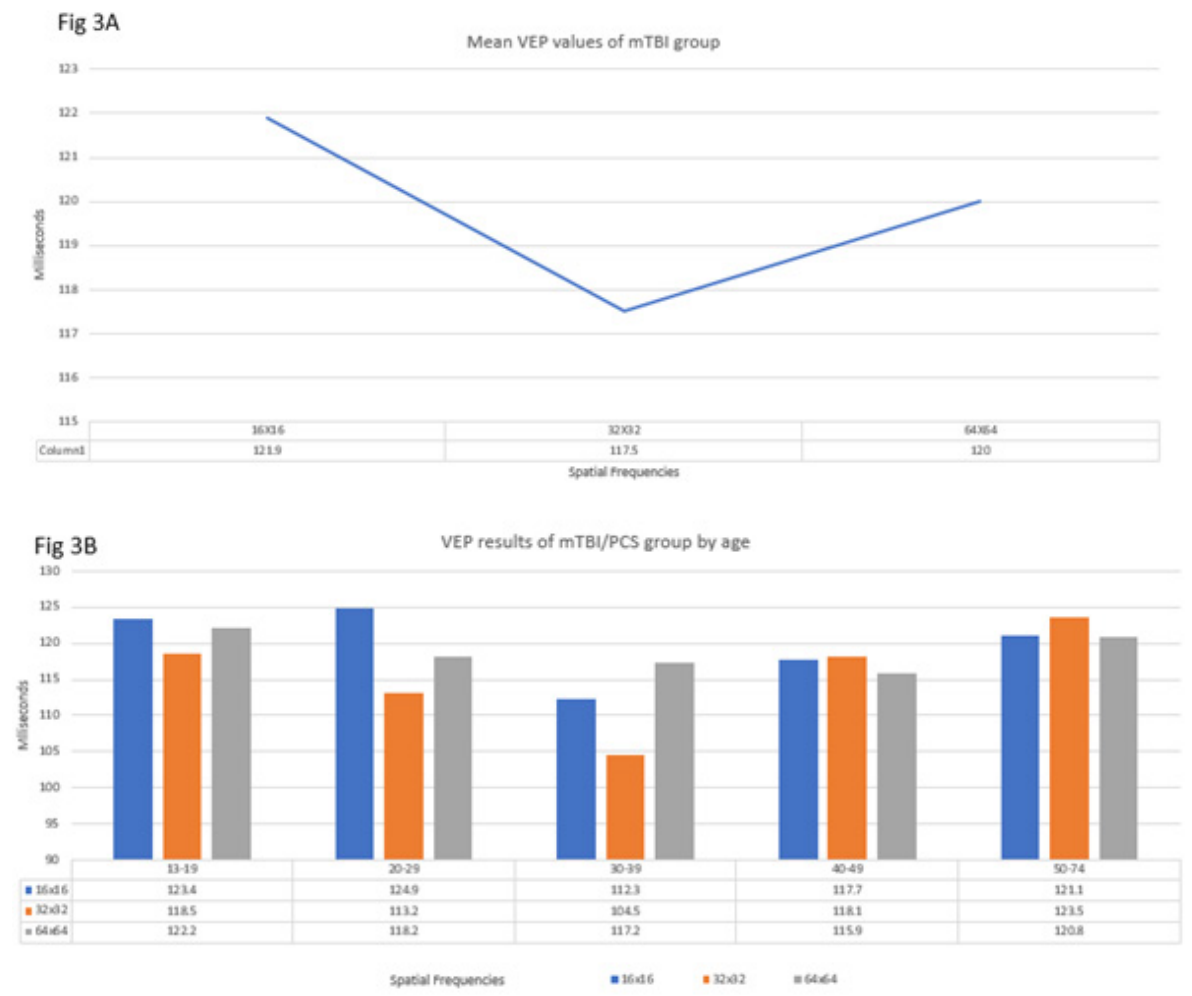

Figure 3: (3A) The VEP responses for the successive spatial frequencies were $121.9 \mathrm{~ms}$ (95\%CI 99.9 to $148 \mathrm{~ms}$ ), $117.3 \mathrm{~ms}$ (95\%CI 95.7 to 150.4$), 120 \mathrm{~ms}$ (95\%CI 58.9 to 142). (3B) VEP results of mTBI/PCS group by age. 

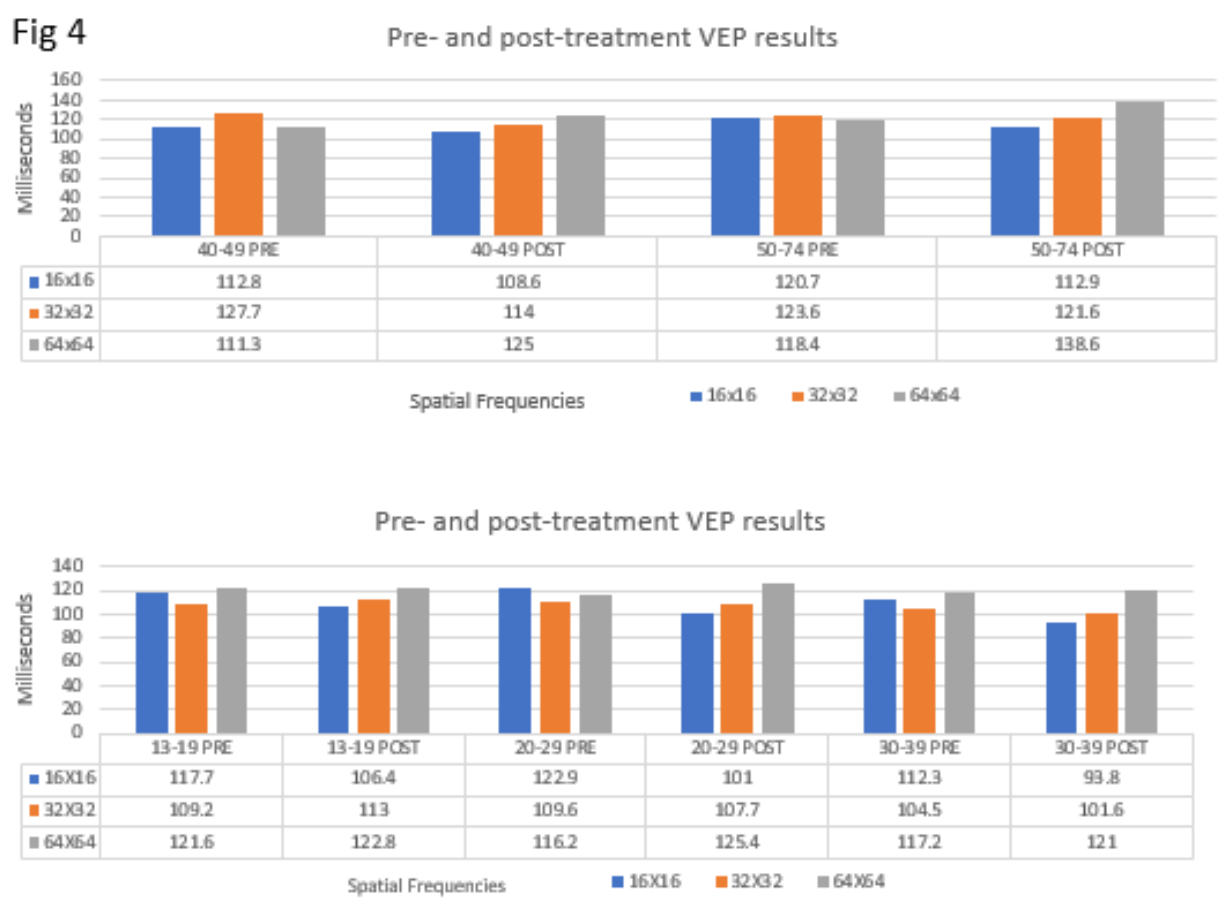

Figure 4: Pre-and Post-treatment mean results. The correlation between all pre-and post-treatment age group at the three spatial frequencies was $16 \times 16(\mathrm{r}=0.00, \mathrm{p}=.999932 \times 32$ ( $\mathrm{r}=0.5788,95 \%$ CI: 0.207 to $0.804, \mathrm{p}=.0048$ and $64 \times 64$ ( $r=.0375,95 \%$ CI: -0.694 to $0.0067, p=.094)$.

\section{Discussion}

Experimental studies have shown that natural scenes, although complex, can be processed by the visual cortex quickly, which indicated that simple and efficient coding processes are involved. Current models of visual perception suggest that the first step of visual perception exists by the extraction of simple features at different SF. The LSF is conveyed by the fast-magnocellular pathways that provides coarse information about a visual stimulus involved in the attentional capture and processing of overall stimulus organization, shape and structure. The HSF conveys finer information about the visual stimulus using the slower parvocellular pathways, that conducts high resolution visual information of the object (e.g. information required for the identification of finegrained edges, borders and color [19].

Kauffmann et al. [20] \& Butler et al. [21] reported that early stage processing is initiated by the rapid LSF and magnocellular pathways (M), which are two important factors effecting processing at higher stages. Deficits in early-stage visual processing significantly predicted higher cognitive and behavioral discrepancies. These findings were confirmed by Butler et al. [21] who reported the existence of early stage visual processing dysfunction in schizophrenia patients by neuroimaging studies, further support our hypothesis that dysfunction within low-level visual pathways involved the thalamocortical radiations [21].

Grossman et al. [9] advocated thalamic injury as a major cause for mTBI symptoms. Sherman [22] proposed the LGN as a useful model for understanding the circuit features found throughout the thalamus. The VEP is not a direct measure of the thalamus, however it plays an important role in the processing of the retinal signal to the primary visual cortex and may indeed play a role in the higher processing stage as proposed by Butler et al. [21] Considerable evidence exists that SF processing takes place in a default coarseto-fine order. Studies using non-human primates suggested that the fast M-pathway LSF access the primary visual cortex and dorsal cortical stream analyzing visual input alerted the parvocellular pathway to primary visual cortex facilitated the slower HSF contribution for recognition of different categories of visual stimuli. Results obtained in this study showed reproducible VEP indications of dysfunction associated with SF processing and its correlation the signs and symptoms associated with mTBI. This is in contrast to the normal group, which showed no signs or symptoms of mTBI and exhibited the expected paradigm of successive SF.

As described by Chang et al. [6] for vision therapy to be effective, motivation, feedback, repetition, sensory-motor mismatch and intermodal integration are necessary components to enhance neuroplasticity changes and following mTBI, function resumes as many neural connections reroutes by creating new neural connections, and these new networks can by-pass the loss of connections. By utilizing top - down visual therapy with recruitment from those cortical areas, VEP assisted in the recovery of function and elimination of symptoms as well as the return of the proper order of LSF latency and HSF latency. 


\section{Conclusion}

The VEP is a practical clinical tool to assess the functional integrity of the retinal signal following a mTBI. The neural foundation of SF processing involved in early stage visual processing is critical for entrance to higher cortical processing. Deficits in early-stage visual processing significantly predict higher cognitive deficits. The VEP testing provided a subcortical level of evaluation when administered properly cannot be faked or misrepresented. The mTBI/PCS individuals showed slower latency for LSF eliciting the signs and symptoms involving scene perception with motion in the retinal image. When the VEP indicated the proper order of latency from coarse to fine then the individuals' symptoms and signs resolved. The VEP test and vision therapy helped close the gap in the evaluation and resolution of mTBI/PCS.

\section{Acknowledgement}

The authors will like to thanks Jessica Watson for proofreading the manuscript.

\section{Conflict of Interest}

The authors have no financial interest related to this study.

\section{Declaration}

The human subjects were treated and consented in agreement with the Declaration of Helsinki Ethical Principles Guidelines.

\section{References}

1. Martin G (2016) Traumatic brain injury: The First 15 Milliseconds. Brain Inj 30: 1517-1524.

2. MacFarlane MP, Glenn TC (2015) Neurochemical cascade of concussion. Brain Inj 29(2): 139-153.

3. Signoretti S, Lazzarino G, Tavazzi B, Vagnozzi R (2011) The pathophysiology of concussion. PM\&R 3: S359-368.

4. Kay T, Harrington DE, Adams R (1993) Definition of mild traumatic brain injury. J Head Trauma Rehabil 8: 86.

5. Rose SC, Fischer AN, Heyer GL (2015) How long is too long? The lack of consensus regarding the post-concussion syndrome diagnosis. Brain Inj 29: 798-803.

6. Chang A, Cohen A, Kapoor N (2013) Top-down visual framework for optometric vision therapy for those with traumatic brain injury | optometric extension program foundation. Optom Vis Perform 1: 48-53.
7. Poltavski D, Lederer P, Cox LK (2017) Visually evoked potential markers of concussion history in patients with convergence insufficiency. Optom Vis Sci 94: 742-750.

8. King NS (2014) A systematic review of age and gender factors in prolonged post-concussion symptoms after mild head injury. Brain Inj 28: 1639-1645.

9. Grossman EJ, Inglese M (2016) The role of thalamic damage in mild traumatic brain injury. J Neurotrauma 33(2): 163-167.

10.King AI, Yang KH, Zhang L, Hardy W (2003) Biomechanics of ligaments: From molecular biology to joint function. In: Frontiers in Biomedical Engineering. Topics in Biomedical Engineering International Book Series. Springer, Boston, USA, pp. 135-147.

11.Little DM, Kraus MF, Joseph J (2010) Thalamic integrity underlies executive dysfunction in traumatic brain injury. Neurology 74(7): 558564.

12.Resch J, Driscoll A, McCaffrey N (2013) Impact test-retest reliability: Reliably unreliable? J Athl Train 48(4): 506-511.

13. Kawata K, Liu CY, Merkel SF, Ramirez SH, Tierney RT, et al. (2016) Blood biomarkers for brain injury: What are we measuring? Neurosci Biobehav Rev 68: 460-473.

14.FDAO of the food and drug administration; FDA authorizes marketing of first blood test to aid in the evaluation of concussion in adults.

15.Banyan Biomarkers.

16.Kou Z, VandeVord PJ (2014) Traumatic white matter injury and glial activation: From basic science to clinics. Glia 62: 1831-1855.

17.Messé A, Caplain S, Paradot G (2011) Diffusion tensor imaging and white matter lesions at the subacute stage in mild traumatic brain injury with persistent neurobehavioral impairment. Hum Brain Mapp 32: 999-1011.

18.Smith DH, Meaney DF (2000) Axonal damage in traumatic brain injury. The Neuroscientist 6: 483-495.

19.Heckenlively JR, Arden GB, Nusinowitz S, Holder GE, Bach M (2006) Principles and practice of clinical electrophysiology of vision. MIT Press, USA.

20.Kauffmann L, Chauvin A, Pichat C, Peyrin C (2015) Effective connectivity in the neural network underlying coarse-to-fine categorization of visual scenes. A dynamic causal modeling study. Brain Cogn 99: 46-56.

21.Butler PD, Zemon V, Schechter I (2005) Early-stage visual processing and cortical amplification deficits in schizophrenia. Arch Gen Psychiatry 62: 495-504.

22.Sherman SM (2007) The thalamus is more than just a relay. Curr Opin Neurobiol 17(4): 417-422.

For possible submissions Click below: 